\section{ROSE SCENT}

\section{Synthesizing monoterpenes Science 349, 81-83 (2015)}

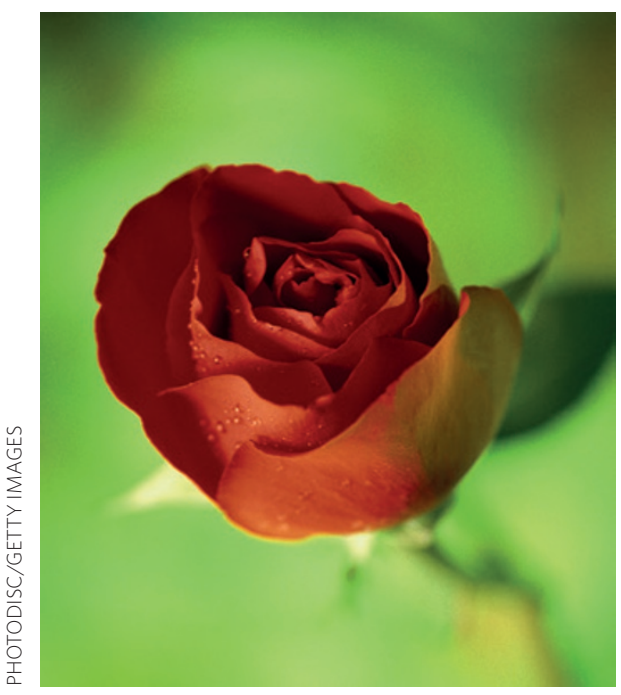

Monoterpenes are the major components in the fragrance of roses and their loss has caused some rose cultivars bred for visual attributes to be odourless. To restore the scent of these roses would require an in-depth understanding of the biosynthetic pathway of monoterpenes. To that end Jean-Louis Magnard and colleagues have identified a Nudix hydrolase, RhNUDX1, responsible for monoterpene biosynthesis and fragrance in roses.

The researchers examined the differences between the transcriptomes of two rose cultivars: the heavily scented Papa Meilland and Rouge Meilland with only a faint scent. The $R h N u D X 1$ gene exhibited the highest differential expression between the two. Furthermore, RhNUDX1 expression correlated with the quantity of monoterpenes in ten rose cultivars, and its mRNA and protein levels correlated with scent emission throughout petal development. Downregulating RhNUDX1 using RNA interference results in reduced monoterpenes. Further analysis co-localized a major quantitative trait locus with RhNUDX1 which explained $76 \%$ of the variation in monoterpene content. Together, these data support the role of RhNUDX1 in monoterpene biosynthesis.

Biochemical assays confirmed the geranyl diphosphate diphosphohydrolase activity of RhNUDX1 in vitro. Using transient expression in tobacco leaves the researchers validated the function of RhNUDX1 in the biosynthesis of geraniol, a monoterpene alcohol, in planta. This may provide a solution for boosting the scent of low-RhNUDX1 roses.

\section{INVASIVE SPECIES}

\section{Trading places}

Global Change Biol. http://doi.org/6cx (2015)

Large numbers of plant species now reside in countries outside of their native range. Although global trade has contributed to the spread of alien species, its impact on the precise movements of plants around the globe has remained unclear.

Hanno Seebens, of the University of Oldenburg, Germany, and colleagues developed a numerical model to assess the influence of bilateral trade on the global

\section{AUXIN SIGNALLING}

\section{Ancestral pathways}

Auxin is central to many aspects of plant development. The molecular mechanism leading to transcriptional changes is well understood, and significant complexity was thought to arise from the high number of components in each functional subfamily, which have overlapping spatiotemporal specificities and work in combination. But now, a plant with an unadorned pathway has been found. John Bowman and colleagues describe auxin signalling in the liverwort Marchantia polymorpha. The conserved but extremely simplified mechanism, with only one orthologue representing each subfamily, makes it the simplest known auxin pathway.

The authors first describe how manipulation of auxin content affects Marchantia development. Auxin sensitivity and plant architecture can be modified by transgenic disruption or overexpression of each signalling component. A severely auxin-insensitive plant almost completely loses its body organization, showing that a minimal set of conserved auxin components is enough to pilot plant development and establish a structured body pattern.

As a descendant of the earliest terrestrial plants, Marchantia is an interesting model for evolutionary studies of signalling pathways, and their importance in land colonization. Moreover, its genetic simplicity, vigorous growth, and the now available tools could soon make this plant a model background system for synthetic biology. movement and distribution of alien plants over the past 60 years, using historical trade, biodiversity and climate data. Their present-day predictions of alien species distributions agree well with observations. The greatest flux of invasive species over this period was from Asia to Europe. However, the establishment of alien species lagged the import of commodities by over 20 years.

Extending their model into the future, the researchers note that large increases in invasive species can be expected in emerging economies over the next two decades, particularly in East Asia and South America. Although climate change may modify the number of invasions over this period, the impact is projected to be small compared with the trade-related increases expected.

\section{RICE AGRICULTURE}

\section{Restricting methane \\ Nature http://doi.org/6f6 (2015)}

Anthropogenic global warming is frequently portrayed as a product of industrialization. However, $7-17 \%$ of the greenhouse gas methane in the atmosphere derives from rice farming. By engineering rice to allocate fewer resources to roots and more to aboveground biomass, Su et al. hope to increase yields while also reducing methane emissions.

Organic molecules exuded from the roots of rice are broken down into methane by microorganisms in the warm waterlogged soils of rice paddies. One way to reduce such methanogenesis is the development of varieties that thrive under drier conditions, but Su et al. took a different approach. They transferred a gene that regulates resource allocation in barley (SUSIBA2) into Nipponbare rice. In barley SUSIBA2 expression is associated with increased starch production and above ground growth and during two years of field trials the engineered rice showed a similar increase in grain yield.

The transgenic plants also had less extensive roots and a reduced community of methanogenic microorganisms in the soil around them. Compared with control plants, methane production was cut by around $90 \%$ before flowering and practically abolished afterwards. This dramatic effect shows that fine-tuning allocation of resources in a crop plant can alleviate the undesirable features of the crop itself and the microbiome that surrounds it.

Written by Anna Armstrong, Jun Lyu, Chris Surridge and Guillaume Tena. 